\title{
Low-gravity L Dwarfs Are Likely More Variable
}

\author{
Stanimir Metchev, ${ }^{1,2}$ Aren Heinze, ${ }^{2}$ Daniel Apai, ${ }^{3}$ Davin Flateau, ${ }^{3}$ \\ Jacqueline Radigan, ${ }^{4}$ Adam Burgasser ${ }^{5}$ Mark Marley ${ }^{6}$ \\ Étienne Artigau, ${ }^{7}$ Peter Plavchan, ${ }^{8}$ and Bertrand Goldman ${ }^{9}$ \\ ${ }^{1}$ The University of Western Ontario, Centre for Planetary Science and Exploration, Canada \\ email: (smetchev@uwo.ca), \\ ${ }^{2}$ Stony Brook University, USA, \\ ${ }^{3}$ The University of Arizona, USA, \\ ${ }^{4}$ Space Telescope Science Institute, USA, \\ ${ }^{5}$ University of California San Diego, Center for Astrophysics and Space Science, USA, \\ ${ }^{6}$ NASA Ames Research Center, USA, \\ ${ }^{7}$ Université de Montréal, Canada, \\ ${ }^{8}$ Missouri State University, USA, \\ ${ }^{9}$ Max-Planck-Institut für Astronomie, Germany
}

\begin{abstract}
In the "Weather on Other Worlds" Spitzer Exploration Science program, we surveyed 44 nearby L3-T8 dwarfs for spot-induced rotational variability. Among single L3-L9.5 dwarfs, we found that $80 \%$ are variable at $>0.2 \%$ in the $3-5 \mu \mathrm{m}$ wavelength range, while $36 \%$ of $\mathrm{T} 0-\mathrm{T} 8$ were variable at $>0.4 \%$. Taking into account viewing angle and sensitivity considerations, both of these findings are consistent with spots being present on $\sim 100 \%$ of L3-T8 dwarfs. Intriguingly, we find a tentative association (92\% confidence) between low surface gravity and high-amplitude variability among L3-L5.5 dwarfs. Although we can not confirm whether lower gravity is also correlated with a higher incidence of variables, the result is promising for the characterization of directly imaged young extrasolar planets through variability.
\end{abstract}

Keywords. brown dwarfs - stars: low-mass - stars:rotation - stars: starspots - stars: variables: general - techniques: photometric

\section{Introduction}

Results from the Weather on Other Worlds (WOW) Spitzer Exploration Science program have been published in Heinze et al. (2013) and Metchev et al. (2015). The former details the data reduction methods and first discoveries, while the latter presents the sample selection, the observing methodology, and a comprehensive discussion of results. In short, the WOW sample comprised 44 targets, of which 39 are single based on ancillary high-angular resolution observations. Each target was observed for 21 consecutive hours in $12 \mathrm{~s}$ exposures: $14 \mathrm{~h}$ in IRAC channel 1 ([3.6]) and $7 \mathrm{~h}$ in IRAC channel $2([4.5])$.

\section{Variability Amplitudes of Low-gravity L3-L5.5 Dwarfs}

Here we focus on the dependence of the observed variability amplitudes with surface gravity in the L3-L5.5 dwarf range. Our sample of 39 unresolved L and T dwarfs contains six individual objects that have been characterized as low- or moderately low-surface gravity dwarfs. Five of these have L3-L5.5 spectral types, and one is the $~ 500$ Myr-old T2.5 dwarf HN Peg B. The tight L3 + L5 binary SDSSp J224953.45+004404.2 — not part of the unresolved sample - also has low surface gravity (Allers et al. 2010): yielding a total of eight low-gravity objects in the complete WOW sample. 


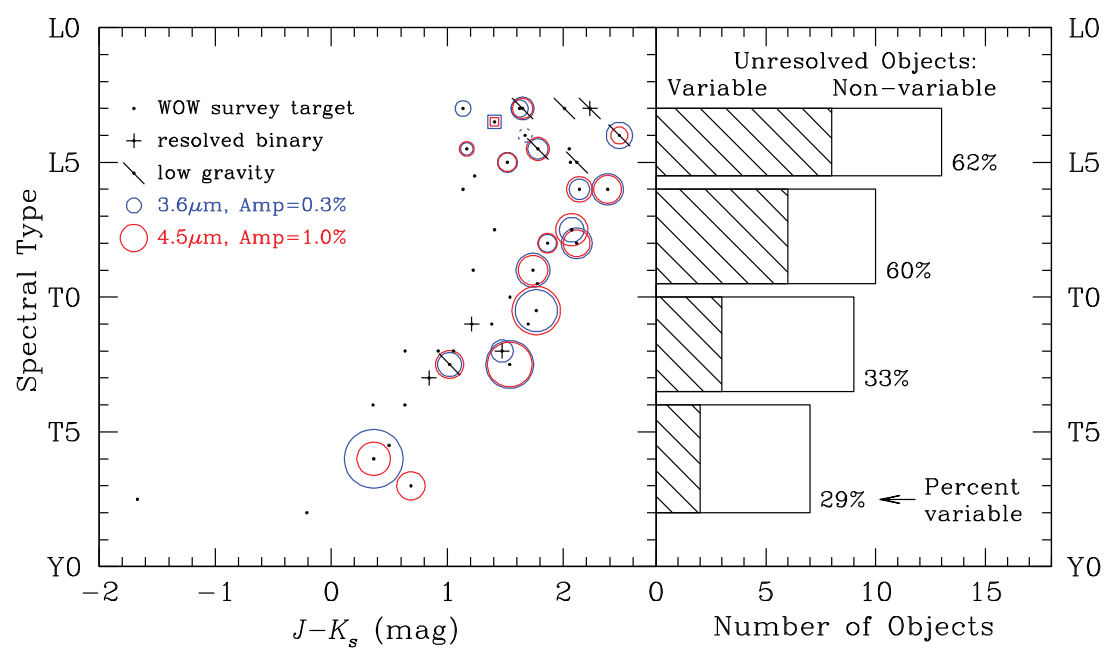

Figure 1. Left: Color, spectral type, and variability distribution of our 44 L3-T8 targets. Circles enclose the variable targets, with the area of the circle proportional to the variability amplitude in the IRAC [3.6] band (blue) or [4.5] band (red). The dashed blue circle encloses object 2MASS J175334518-6559559 (L4), which displays only a linear trend at [3.6], and does not have a well defined amplitude. The previously known magnetically active L3.5 dwarf 2MASSW J0036159+182110 is variable and shown with concentric squares. Known tight binaries are marked with + , and are plotted at their systemic spectral type and colour. Inclined bars denote low-gravity objects, including six L3-L5 dwarfs (one a close binary) and the T2.5 dwarf HN Peg B. Right: Distribution and frequency of [3.6] or [4.5] variability of the 39 objects in our unresolved sample, excluding the previously known magnetically active L3.5 variable 2MASSW J0036159+182110.

The variability fraction among the putative low-gravity L3-L5.5 dwarfs is $3 / 7$ or $3 / 5$, depending on whether the individual components of the non-varying binary SDSSp J224953.45+004404.2 are counted separately, or whether it is altogether excluded from the sample. Within the statistical uncertainties, this is indistinguishable from the fraction of variables among the high-gravity objects in the L3-L5.5 bin: 5/8.

However, we do detect a tentative correspondence between amplitude and surface gravity among the set of eight L3-L5.5 dwarfs that are variable. The three L3-L5.5 variables that show signatures of low gravity also have the highest [3.6]-band amplitudes in the L3-L5.5 bin (Fig. 1): 2MASS J16154255+4953211 (L4 $\beta$ ), 2MASSW J2208136+292121 $(\mathrm{L} 3 \gamma)$, and 2MASS J18212815+1414010 (L4.5). The latter two objects also have the highest bin amplitudes at [4.5]. A consideration of all possible ways to choose three objects from eight shows that the three low-gravity variables would have the highest amplitudes among the eight variables in the L3-L5.5 bin in $\frac{3}{8} \frac{2}{7} \frac{1}{6}=1.8 \%$ of cases. That is, the result might appear $98.2 \%$ significant.

More generally, we would have likely considered any outcome that includes the amplitudes of the three low-gravity L3-L5.5 dwarfs among the top half in the bin. We also need to incorporate the four low-gravity L3-L5.5 dwarfs - including the individual near-equal flux components of the L3 + L5 binary SDSSp J224953.45+004404.2 - that are not detected as variables. Otherwise, the exclusion of censored data could bias our conclusion. We test the significance of the result by combining all detections and non-detections in a Monte Carlo approach (Metchev et al. 2015). To account for the diminished sensitivity to variations from either of the components of the L3 + L5 binary, we count it as two individual objects that are half as bright. We consider as positive any outcome that includes at least three detected low-gravity L3-L5.5 variables, with [3.6] or [4.5] amplitudes 
all in the top half of the L3-L5.5 bin. We find that this scenario arises at random in $8 \%$ of our simulations. That is, the association between low surface gravity and enhanced variability amplitude is $92 \%$ significant.

We note that the three high-amplitude L3-L5.5 variables are also redder compared to the lower-amplitude ones (Fig. 1). That is, the higher variability amplitudes may be related to higher dust content in the atmospheres. Nonetheless, low surface gravities in ultra-cool dwarfs correspond strongly with redder photospheric colours. Hence, while low gravity by itself may not be the reason for the higher variability amplitudes, it is still likely correlated with them.

\section{Conclusion}

While we cannot conclude that low surface gravity leads to higher incidence of detectable variability, we find that low gravity and/or dustiness may be correlated with higher 3-5 $\mu \mathrm{m}$ amplitudes among variable L3-L5.5 dwarfs. This is promising for measuring the rotation periods and cloud compositions of directly imaged extrasolar giant planets through variability.

\section{References}

Allers, K. N., Liu, M. C., Dupuy, T. J., \& Cushing, M. C. 2010, ApJ, 715, 561

Heinze, A. N., et al. 2013, ApJ, 767, 173

Metchev, S. M. et al. 2015, ApJ, 799, 154 